\title{
Beyond the obvious cranial abnormalities in fetal MMC. (Mini-commentary on BJOG-20-1008.R1)
}

\author{
Thierry Huisman ${ }^{1}$ \\ ${ }^{1}$ Texas Children's Hospital, Department of Radiology
}

September 11, 2020

Mini-commentary on BJOG-20-1008.R1: Cranial findings detected by second trimester ultrasound in fetuses with myelomeningocele: a systematic review

\section{Title: Beyond the obvious cranial abnormalities in fetal MMC}

Author information:Thierry A.G.M. Huisman, Department of Radiology, Texas Children's Hospital Baylor College of Medicine 6701 Fannin Street, Suite 470 Houston, TX 77030 email: huisman@texaschildrens.org

I write with pleasure a commentary to the excellent review by Dr. Kunpalin and colleagues on the spectrum and frequency of cranial findings that may be encountered by second trimester ultrasound in fetuses diagnosed with non-skin covered spinal dysraphias (BJOG xxxx). The early and adequate recognition of associated or presumed non-associated/incidental imaging findings have a significant impact on decision making of intrauterine corrective surgical procedures. The association between a spinal dysraphia and the occurrence of a Chiari 2 malformation $(\mathrm{C} 2 \mathrm{M})$ are linked to the mechanical consequences of a venting/leakage of cerebro-spinal fluid (CSF) from the developing caudal embryonic rhombencepalic and metencephalic vesicles during early fetal life. This unified theory does not explain the entire spectrum of anatomical abnormalities that may be observed on imaging. Additional complex molecular genetic and environmental factors also play a role. The authors summarize 14 obvious sonographic findings that may be detected during the second trimester. They reference that "About one-third are in the C2M spectrum and can be attributed to the mechanical consequences of a CSF leakage. Some abnormalities may not be obviously explained by the CSF leakage but their frequency indicate that they are someway linked". I completely agree with this conclusion. Careful analysis of high-resolution anatomical and functional postnatal imaging studies have shown that the brainstem, cerebellum and cerebrum are highly abnormal beyond the obvious morphological distortion. Diffusion tensor imaging (DTI) studies revealed an abnormal micro-structural neuro-architecture and anomalous maturation of multiple white matter tracts (Semin Ultrasound CT MR. 2016 Apr;37(20):129-142). For example, qualitative DTI analysis and fiber tractography show a reduction in size of the transverse pontine fibers as the most likely explanation of pontine hypoplasia and anterior displacement of the dentate nuclei. DTI also shows a decrease in the fiber density of the middle cerebellar peduncles and cerebellar hypoplasia. Furthermore next to the obvious corpus callosum (CC) hypo-dysplasia spectrum which is likely secondary to a global white matter defect, in about $60 \%$ of children with $\mathrm{C} 2 \mathrm{M}$ a dorsal callosal ridge is seen representing an interhemispheric dysplastic small white matter bundle running over the dorsal CC. Finally the increased incidence of true malformations of cortical development (e.g. polymicrogyria), subependymal gray matter heterotopias, malrotated and laterally displaced hippocampi, accessory lobes, defined as gyri protruding into the posterior interhemispheric cistern (20\% of patients), and an anomalous, possibly duplicated massa intermedia confirm that the anomalous morphologically less obvious or presumed non-associated malformations are secondary to the anomalous CSF-hemodyamics and pressure dynamics interacting/disrupting the various components of the complex programmed brain development (e.g. neuronal migration, intracortical laminar organization, myelination/sulcation, axonal guidance). Finally, the genetic association between the 
spinal dysraphia and identified intracranial malformations is consequently much more complex and linked than initially presumed. With ongoing improvements in the diagnostic sensitivity and specificity of prenatal imaging more detailed components of this complex malformation complex will become "detectable" at an earlier phase of pregnancy and a careful correlation of these findings with clinical outcome appears to be a sine qua non for parental counselling and surgical decision making.

No disclosures: A completed disclosure of interest form is available to view online as supporting information. 